\title{
Improving the realization of individualized prostheses by optical measurement of mandibular kinematics
}

To improve the dental prostheses of their patients, more and more practitioners make individualized prostheses by measuring mandibular kinematics.

At the IDS 2019 I had the opportunity to examine several devices which measure mandibular kinematics. Out of all these, I have chosen to present to you the new measurement system produced by the renowned company Zebris Medical $\mathrm{GmbH}$. While at the company stand, Eng. Wolfgang Brunner, one of the owners of the company, made a very detailed presentation of the Optic Jaw Motion Analyzer.

This system extends Zebris'US JMA systems with state-ofthe-art optical sensor technology. The analyzer is made of a handy table stand with an inductive charging station and face bow with a lower jaw sensor.

The face bow is easily mounted by adjusting the nasion support, sliding porion earpieces and spring-mounted side arms. The jaw sensor is magnetically fixed onto the paraocclusal or occlusal attachments to the lower teeth. The bite fork establishes the exact relationship between the movement data in the measuring system and the surfaces of the teeth scanned by the model and intraoral scanner.

The system has the possibility to cover a wide range of applications from the functional analysis, the analysis of the position of the condyles, the determination of the neuromuscular jaw relationship, to the creation of functional dental restorations, the planning, documentation and monitoring of the rehabilitation of the stomatognathic system.

The system has the ability to optionally operate with a USB interface or over Wi-Fi. The JMAOptic-system has a modular and intuitive analytical software WINJAW+ which includes a database, the basic module and an export function. Real patient data or settings of virtual articulators can be transferred to the external CAD systems by exporting into XML format.

In conclusion, the optical JMA system includes:

- an electronic face bow with camera and infrared modules;

- a T-pointer;

- a lower jaw sensor:

- attachments;

- an analytical WINJAW + software with basic module articulator and data export:

- a user manual;

- a carry case.

With the experience gained by using the Zebris' US JMA System since 2008, I look forward to receiving this new evaluation device and to sharing my experience with this system once the JMA Optic series are produced.

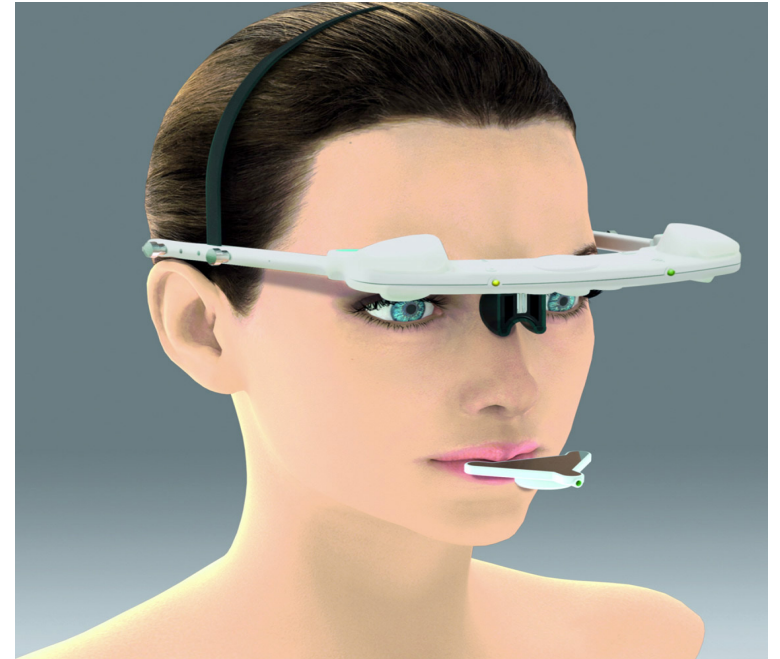

The Optic Jaw Motion Analyser (Zebris Medical GmbH, D-88316 Isny, Germany) https://www.zebris.de/en/dental/products-solutions/

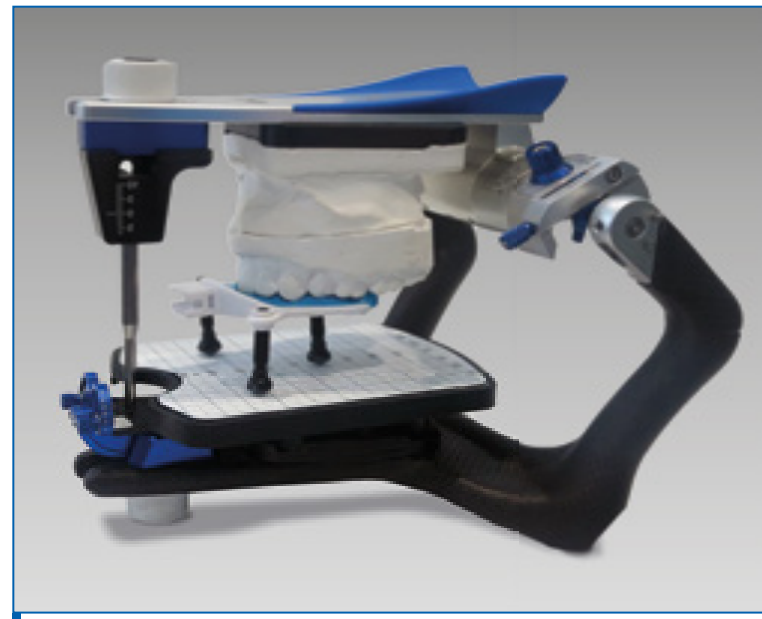

Transfer stand for transferring the maxillary position to mechanical articulators using the bite fork

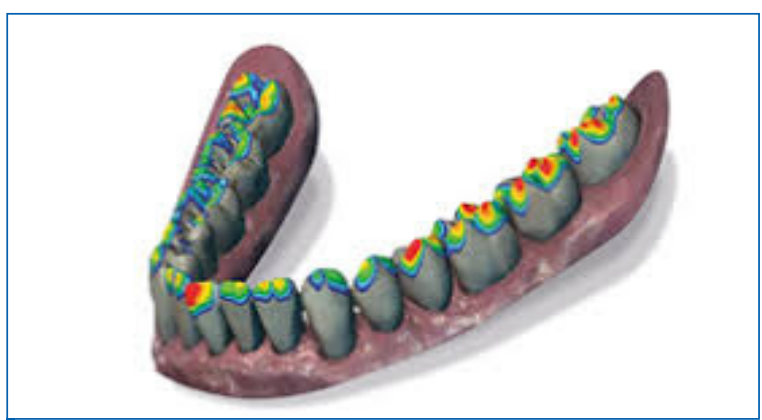

The system is ready to visualize the static and dynamic contact situation.

Florin - Eugen Constantinescu DMD, PhD Student Editorial Director, Product News 